\title{
Liberalisation du Transport Maritime et Developpement des Ports Ivoiriens
}

\author{
Gnakan Maguil Mouto, \\ Jean Tapé Bidi, \\ Université Félix Houphouët-Boigny, \\ Institut de Géographie Tropicale, Cote d'Ivoire
}

\section{Résumé}

En 1951 et 1970, avec la construction du port d'Abidjan puis de San Pedro, la Côte d'Ivoire se dote d'infrastructures portuaires modernes. Cellesci sont alors gérées par l'État qui était le principal acteur de leur développement. Affaiblit par la crise des années 1980, l'État ivoirien, sous la pression des bailleurs de fonds, s'est désengagé des activités de production y compris de ceux du secteur maritime et portuaire qui peinaient à amorcer véritablement leur développement. Les infrastructures et équipements portuaires lorsqu'ils n'étaient pas vieillissants, étaient simplement inexistants faute d'investissement adéquats. L'ouverture aux capitaux privés, une politique vivement encouragée par la Banque Mondiale et le Fond Monétaire International s'est présentée comme unique possibilité pour tirer les ports ivoiriens de leur état de sous-développement. L'objectif assigné à cette étude est d'analyser les effets de la libéralisation sur le développement des ports d'Abidjan et de San Pedro. La méthode retenue pour aboutir aux résultats s'inscrit dans une approche qualitative. Cette démarche intègre la recherche documentaire, l'observation directe sur le terrain et l'interview de personnes ressources au moyen d'un guide d'entretien. Les résultats obtenus à l'issue de l'analyse des données révèlent que les opérateurs privés sont au premier plan dans le processus de développement des ports ivoiriens. Ils agissent positivement sur tous leurs indicateurs de performance portuaire. Ce qui contribue à leur attractivité. En revanche, ils mettent en exergue le risque réel d'un transfert d'un monopole public à celui d'un monopole privé.

Mots clés: Ports ivoiriens, Libéralisation, Transport maritime, Développement 


\title{
Liberalization of Maritime Transport and Development of Ivorian Ports
}

\author{
Gnakan Maguil Mouto, \\ Jean Tapé Bidi, \\ Université Félix Houphouët-Boigny, \\ Institut de Géographie Tropicale, Cote d'Ivoire
}

\begin{abstract}
In 1951 and 1970, with the building of the port of Abidjan and San Pedro that the Cote d'Ivoire has acquired itself the modern infrastructures harbour. They have then managed by the State that was the main actor of their development. Weakened by the the crisis of $80 \mathrm{~s}$, the Ivorian government, under the pressure of financial sponsors is free itself from some activities of production including those of navy sector and harbour who were struggling to really start their development. The ports of Abidjan and San Pedro have trouble priming really their development. The infrastructures and harbour equipments when they are not new are simply inexistant because of the lack of adequate investments. The openess to private capital, a policy strongly encouraged by World Bank and the International Monetary Fund presented itself as the only possibility to pull the Ivorian ports from their underdevelopment state. The objective related to this study is to analyze the effects of the liberization on the development of the ports of Abidjan and San Pedro. The best method consists in attaining the results related to a qualitative and quantitative approach. This method integrates the documentary research, the direct view on the field and the interview of resource persons in a way of guide talk. The results come from the analysis of datas reveal that the private operators are at the first level in the process for the development of the Ivorian ports. They positively react on all their indicators of harbour performances. What contributes to their attractivity. On the other hand, they highlight the real risk of a transfer of the public monopoly to those of a private monopoly.
\end{abstract}

Keywords: Abidjan, San Pedro, Cote d'Ivoire, Liberalization, Maritime transport, Development

\section{Introduction}

La Côte d'Ivoire, lors de son accession à l'indépendance, fait le choix de poursuivre le système déjà instauré par la métropole, à savoir une économie 
basée sur la production et l'exportation de matières premières. Cette période, jusqu'en 1979, est marquée par un coût élevé du binôme café-cacao, matières sur lesquelles repose l'économie ivoirienne. Elle est aussi caractérisée par une prospérité économique. L'État se fortifie et opte pour une gestion centralisée ; il intervient dans tous les domaines d'activités.

Au début des années 1980, on assiste à une baisse du prix du cacao et du café. Pourtant, la croissance économique de la Côte d'Ivoire est dépendante de ces deux cultures. C'est pourquoi le FMI et la Banque Mondiale interviennent pour ne pas laisser faillir totalement 1'Etat. C'est le temps de l'ajustement structurel. L'État se « désengage » et le secteur privé, soutenu par les instances internationales prend le relais (OSMONT, 1996). Ce premier programme d'ajustements réalisé en 1981 n'a pas permis de résorber les effets de la crise économique. En 1994, un autre programme d'ajustement fut initié. Il touchait à la levée des mesures protectionnistes que l'Etat accordait aux armements nationaux en échange d'un prêt financier. En réalité la BM exigeait la libéralisation du transport maritime. Ce que fut la Côte d'Ivoire. Depuis lors, la physionomie du secteur maritime et portuaire ivoirien a profondément changé. On assiste à l'arrivée de nouveaux acteurs et à l'implication du secteur privé dans certains domaines clés longtemps tenus par le secteur public.

En dépit de ces données sur la libéralisation du transport maritime en Côte d'Ivoire, force est d'admettre l'insuffisance d'informations circonscrites aux effets de cette politique sur l'économie portuaire ivoirienne. Cette étude propose d'analyser les effets de l'ouverture du marché du transport maritime sur le développement des ports d'Abidjan et San Pedro.

\section{Methodologie}

La méthode retenue pour cette étude s'inscrit dans une approche qualitative. Elle intègre trois points essentiels à savoir, la recherche documentaire, l'observation directe sur le terrain et l'interview de personnes ressources au moyen d'un guide d'entretien.

La recherche documentaire a consisté dans un premier temps à inventorier la documentation en rapport avec le sujet porté à notre analyse, à les analyser puis à les grouper par type. Les lectures ont porté sur la libéralisation du transport maritime de manière générale. Plus particulièrement, la fouille documentaire insiste sur les effets de cette politique sur la macro économie des États africains. Elle a également renseigné sur l'impact de libéralisation sur l'emploi en milieu portuaire, la gouvernance des ports et sur le développement de ces entités. Cette littérature nécessaire à la collecte des données s'est appuyée sur des rapports d'activités et des documents techniques (sur les concessions et les projets de développement) des ports d'Abidjan et de San Pedro. Des ouvrages généraux, des articles et des thèses viennent compléter cette bibliographie. Au total, la consultation de 
travaux similaires réalisés ailleurs s'est avérée intéressant pour percevoir les outils et les points utiles pour une évaluation efficiente des incidences de la politique de la libéralisation du transport maritime sur le développement des ports en général et des ports ivoiriens en particulier.

Quant à l'observation directe, elle a été le cadre adéquat ayant permis de nous imprégner des réalités du terrain. Des excursions aux ports d'Abidjan et de San Pedro ont permis d'apprécier la diversité d'opérateurs qui y séjournent. Nous avons pu observer les équipements portuaires ainsi que les travaux d'aménagement en cours au port d'Abidjan notamment (travaux du remblaiement pour la construction d'une zone industrielle, élargissement et approfondissement du canal de Vridi et construction du second terminal à conteneurs).

Enfin, des interviews ont eu lieu avec des responsables des structures suivantes : la Direction générale des affaires maritimes et portuaires, le port autonome d'Abidjan, le Conseil burkinabé des chargeurs, les Entrepôts maliens en Côte d'Ivoire, Necotrans, MSC, Bolloré à Abidjan et CMA CGM du côté de San Pedro. Les discussions ont porté sur le processus d'intégration des opérateurs privés dans le milieu maritime et portuaire ivoirien et de leur rôle effectif dans leurs processus de développement. L'intérêt que les ports d'Abidjan et de San Pedro accordent à ces derniers a constitué un pan important de nos échanges.

\section{Resultats}

1. La Situation des Ports Ivoiriens Avant la Liberalisation du Transport Maritime en Cote d'Ivoire

Avant l'avènement de la libéralisation, les autorités publiques ivoiriennes étaient au cœur de la gestion des activités maritimes et portuaires. Cet interventionnisme de l'État s'est traduit par l'élaboration d'une politique forte et par la mise en place de nombreuses structures qu'il gérait lui-même : l'État jouait ainsi un rôle majeur dans les activités maritimes et portuaires.

\subsection{Les instruments de l'intervention de l'État dans la gestion portuaire en Côte d'Ivoire}

Les transports maritimes constituent indéniablement un secteur d'activité essentiel pour un pays à économie extravertie comme la Côte d'Ivoire. Conscient du rôle stratégique de cette activité pour l'essor de leur territoire, les autorités de l'époque ont élaboré une politique maritime nationale. Elle s'appuyait sur un arsenal juridique et des structures opérationnelles à même de garantir le transport par voie de mer. Il s'agit entre autres de la SITRAM $^{1}$ et de la SIVOMAR ${ }^{2}$. La politique maritime ivoirienne

\footnotetext{
${ }^{1}$ Société ivoirienne de transport maritime

${ }^{2}$ Société ivoirienne de navigation
} 
allait au-delà du seul aspect de la création d'une flotte marchande et de structures auxiliaires. Elle s'étendait aussi aux domaines de la formation avec l'Académie Régionale des Sciences et Techniques de la Mer (ARSTM) et de la recherche avec l'Institut de Documentation, de Recherches et d'Études Maritimes (IDREM). Le Ministère de la marine marchande, créé par la volonté de l'l'État a été la pierre d'angle de la politique maritime de la Côte d'Ivoire. Elle a été l'organe à partir duquel toutes les actions maritimes et portuaires ont été planifiées ; le centre d'impulsion des décisions majeures relatives à la marine marchande ivoirienne.

La Côte d'Ivoire, ancienne colonie française a hérité de sa tradition régalienne. Cette situation faisait de l'État ivoirien le point focal de l'appareil administratif, politique et économique. Ce dirigisme se manifestait par la forte implication de l'État dans le secteur primaire, secondaire que tertiaire. Dans les milieux maritimes et portuaires cette présence publique se ressentait également. Outre sa fonction de gestionnaire administrative des places portuaires d'Abidjan et de San Pedro, il y jouait le rôle de principal animateur par son intervention dans les différents domaines d'activités qui les constituent (tableau 1).

Tableau 1 : Domaines d'intervention des autorités publiques aux ports d'Abidjan et San Pedro avant 1992

\begin{tabular}{|l|c|c|}
\hline \multirow{2}{*}{ Services aux navires } & \multicolumn{2}{|c|}{ Dénomination ports } \\
\cline { 2 - 3 } & PAA & PASP \\
\hline Attribution des postes à quai & $\mathrm{X}$ & $\mathrm{X}$ \\
\hline Contrôle de la navigation & $\mathrm{X}$ & $\mathrm{X}$ \\
\hline Consignation & $\mathrm{X}$ & $\mathrm{X}$ \\
\hline Pilotage & $\mathrm{X}$ & $\mathrm{X}$ \\
\hline Remorquage & $\mathrm{X}$ & $\mathrm{X}$ \\
\hline Lamanage & $\mathrm{X}$ & $\mathrm{X}$ \\
\hline Réparation navale & - & - \\
\hline Fourniture d'eau & $\mathrm{X}$ & $\mathrm{X}$ \\
\hline Avitaillement & - & - \\
\hline Dragage, récupération de déchet & $\mathrm{X}$ & $\mathrm{X}$ \\
\hline Recouvrement des droits de port & $\mathrm{X}$ & $\mathrm{X}$ \\
\hline Contrôle de sécurité & $\mathrm{X}$ & $\mathrm{X}$ \\
\hline Sécurité incendie & $\mathrm{X}$ & $\mathrm{X}$ \\
\hline
\end{tabular}




\begin{tabular}{|l|c|c|}
\hline \multicolumn{3}{|c|}{ Services à la marchandise } \\
\hline Transit & $\mathrm{X}$ & $\mathrm{X}$ \\
\hline Manutention & $\mathrm{X}$ & $\mathrm{X}$ \\
\hline Dédouanement & Dénomination ports \\
\hline Services aux navires & PAA & PASP \\
\hline Contrôles douaniers & - & - \\
\hline Contrôles sanitaires & - & - \\
\hline Transport routier & - & - \\
\hline Transport ferroviaire & - & - \\
\hline Stockage & - & - \\
\hline Suivi des marchandises dangereuses & $\mathrm{X}$ & $\mathrm{X}$ \\
\hline \multicolumn{1}{|c|}{ Infrastructures et superstructures } \\
\hline Hydrographie-dragage & $\mathrm{X}$ & $\mathrm{X}$ \\
\hline Entretien des quais et terre-pleins & $\mathrm{X}$ & $\mathrm{X}$ \\
\hline Gestion de l'outillage & $\mathrm{X}$ & $\mathrm{X}$ \\
\hline Étude et travaux d'extension & $\mathrm{X}$ \\
\hline
\end{tabular}

Source: PAA, PASP et Mouto, 2014

$\mathrm{X}$ : Ports autonomes

- : Autres acteurs publics/privés

L'examen du tableau montre l'étendue du champ d'action de l'autorité publique. En effet, avant l'année 1992 qui marque le début du processus de libéralisation des transports maritimes, l'État ivoirien est très présent dans tous les compartiments des opérations maritimes et portuaires. Les trois grands secteurs portuaires, à savoir les services aux navires, les services à la marchandise ainsi que la réalisation des infrastructures et superstructures étaient entièrement couverts par des entreprises publiques.

\subsection{Les conséquences de l'interventionnisme de l'État dans la gestion des ports ivoiriens}

Le fait pour l'État d'intervenir dans l'ensemble des services maritimes et portuaires ivoiriens présentait des intérêts à bien des égards. S'était pour lui le moyen d'exercer un contrôle sur la logistique. Il pouvait ainsi organiser à souhait toutes les expéditions nécessaires au bon fonctionnement de son 
commerce extérieur sans vraiment dépendre d'un opérateur quel qu'il soit ou sans tenir compte du coût de manutention qui pouvait avoir des effets négatifs sur la compétitivité des produits exportés depuis Abidjan et San Pedro. Les retombées pour les pouvoirs publics se situaient à la fois au niveau économique, social et politique.

Ainsi, au plan socioéconomique l'exploitation des terminaux portuaires par les pouvoirs publics était doublement avantageuse pour l'État ivoirien. Elle lui permettait de réaliser des opérations de manutention à moindre coût et des réaliser des bénéfices sur les prestations offertes aux autres acteurs qui le sollicitaient. Les coûts additionnels liés au poste fret se voyaient ainsi réduits de manière conséquente. Par ailleurs, la SITRAM International Shipping Agencies (SISA), en assurant la consignation de plusieurs navires qui desservaient de manière régulière les ports ivoiriens et en contrôlant plus de $80 \%$ du fret ivoirien, garantissait à l'État des devises à travers les taxes payées par les autres entreprises. En se référant au tableau 2 ci-dessous, il est donné de voir qu'entre 1980 et 1994, période de la participation de la Côte d'Ivoire au transport maritime, le fret payé au titre de l'exportation par mer était en moyenne de 51 milliards. Ce chiffre est en dessous des 75 milliards en moyenne payés avant 1980, soit une baisse de $32 \%$ et aux 100 milliards payés après 1994, soit le double du fret payé par la Côte d'Ivoire quand elle participait aux conférences maritimes.

Tableau 2 : Nomenclature des frets maritimes en fonction du trafic maritime

\begin{tabular}{|l|c|c|c|}
\hline Périodes & $\mathbf{1 9 7 0 - 1 9 7 9}$ & $\mathbf{1 9 8 0 - 1 9 9 4}$ & $\mathbf{1 9 9 5 - 2 0 0 2}$ \\
\hline Caractéristiques & Libre-échange & $40-40-20$ & Libéralisation \\
\hline Quantités exportées (1 000 T) & 20837 & 26532 & 54395 \\
\hline Moyenne annuelle (1 000 T) & 4167 & 4422 & 6799 \\
\hline Fret moyen reçu (milliards) & 25 & 87 & $11^{6}$ \\
\hline Fret payé (milliards) & 75 & 51 & 100 \\
\hline Situation nette par an (milliards) & -50 & 36 & -86 \\
\hline
\end{tabular}

Source: Plan quinquennal 75-80, Lettre de Conjoncture $\mathrm{N}^{\circ} 12$

(mars 2002 pour la période allant de 1990 à 2002) et Guessan Bi, 2004

L'existence de sociétés maritimes a favorisé la création d'emplois et constituait un débouché pour les nombreux élèves officiers et sous-officiers sortis de l'académie régionale des sciences et techniques de l'ARSTM. D'un point de vue politique ces structures réduisaient de manière considérable la trop forte dépendance de la Côte d'Ivoire vis-à-vis des compagnies étrangères pour la réalisation de ses échanges commerciaux. 
Cependant, l'action de l'Etat n'a pas que des points positifs. La révolution des transports maritimes induite par la conteneurisation lève un coin de voile sur les limites de son intervention. Pour s'adapter aux nouvelles caractéristiques des navires en circulation, les ports sont appelés à subir de profondes transformations tant au niveau de leurs structures que de leurs équipements. La taille des navires et le volume des marchandises imposent des projets grandioses qui représentent de vrais défis économiques pour les Etats.

Or, un pays comme la Côte d'Ivoire, pris dans la spirale de récession économique des années 1980 ne peut prétendre à un tel luxe. L'État, sous la contrainte des bailleurs de fonds, s'est donc désengagé de plusieurs secteurs d'activités, y compris celui des ports et transports maritimes. Les entreprises publiques de cette sphère n'étant pas préparées au retrait de leur principal bailleur, ont connu des difficultés qui ont conduit plus tard, pour les uns, à leur disparition et pour les autres, à la redéfinition de leur feuille de route. A partir de 1990, face à la situation et devant la pression des bailleurs de fonds, l'Etat a entamé une série de réforme en vue de faciliter le développement d'un partenariat public/privé

\section{Le Développement d'un Partenariat Entre le Public et le Prive}

Plus qu'un simple besoin, le développement d'un partenariat entre les représentants des autorités portuaires d'Abidjan et San Pedro et les opérateurs privés s'annonce comme un impératif tant les exigences en termes de modernisation et de transfert d'un savoir-faire sont énormes. Mais la signature de ces accords publics/privés suppose le changement de certaines habitudes. C'est la raison pour laquelle les autorités en charge de la gestion des ports d'Abidjan et de San Pedro ont approuvé de nouvelles réformes. Ces dernières ont consisté à scinder les tâches qui leur incombaient auparavant. On assiste alors à la séparation de la fonction de gestion de celle des opérations d'exploitation. Timide au départ, la participation des acteurs privés aux activités portuaires à Abidjan et San Pedro a atteint un niveau élevé à telle enseigne que la quasi-totalité des opérations commerciales leur a été légué. Et le régime concessif est la méthode par excellence retenue pour l'incursion des opérateurs privés dans les activités portuaires en Côte d'ivoire.

\subsection{Le régime concessif, cadre institutionnel du partenariat public et privé portuaire en Côte d'Ivoire}

En Côte d'Ivoire, les concessions sont utilisées dans le cadre des réformes des ports. Elles visent à accroître la participation du secteur privé dans la gestion portuaire afin de promouvoir une plus grande efficacité et réduire les coûts commerciaux. Elles font interagir les autorités concédantes, à savoir le PAA et le PASP et les opérateurs privés qui sont les concessionnaires. Les objets de la concession sont aussi divers que le sont les 
concessionnaires eux-mêmes. Il s'agir notamment de concessions d'installations existantes comme ce fut le cas du terminal à conteneurs de Vridi ou de projets nouveaux comme en témoigne le terminal à conteneurs 2 d'Abidjan - TC2 d'Abidjan -. Ainsi, les concessions portuaires à Abidjan et San Pedro portent principalement sur les terminaux spécialisés et sur certaines activités. On parle alors de concession partielle. Le processus Construire, Exploiter et Transférer (CET) connu sous le sigle anglais BOT qui signifie Build, Operate, Transfer est lui le modèle de contrat de concession privilégié par les autorités des deux ports ivoiriens. Il permet de développer le partenariat que les autorités des ports de San Pedro et d'Abidjan entreprennent depuis quelques années avec le secteur privé. En conséquence, depuis le 23 octobre 2003, année de la signature de la convention de concession pour la gestion et l'exploitation du terminal à conteneurs de Vridi, ce type d'accord de partenariat s'est aussitôt répandu aux installations et services du port d'Abidjan. Il a atteint en 2008 le port de San Pedro avec la mise en concession de son terminal à conteneurs à la société italo-suisse Mediterranean Shipping Company (MSC). Le tableau 3 établi dans l'ordre chronologique les installations et services portuaires d'Abidjan et San Pedro concédés à des opérateurs privés.

Tableau 3 : Les services et activités portuaires concédés aux ports d'Abidjan et San Pedro

\begin{tabular}{|c|c|c|c|c|}
\hline Convention de concession & $\begin{array}{l}\text { Autorité } \\
\text { concédant }\end{array}$ & Concessionnaire & $\begin{array}{l}\text { Année de } \\
\text { concession }\end{array}$ & $\begin{array}{l}\text { Durée de } \\
\text { concession }\end{array}$ \\
\hline $\begin{array}{l}\text { Gestion et exploitation du Terminal } \\
\text { à conteneurs de Vridi }\end{array}$ & $\begin{array}{l}\text { Port Autonome } \\
\text { d'Abidjan }\end{array}$ & Abidjan Terminal (ATL) & 2004 & 2019 \\
\hline $\begin{array}{l}\text { Aménagement et exploitation du } \\
\text { Terminal roulier d'Abidjan (TERA) }\end{array}$ & $\begin{array}{l}\text { Port Autonome } \\
\text { d'Abidjan }\end{array}$ & $\begin{array}{l}\text { Terminal Roulier d'Abidjan } \\
\text { (TERA) }\end{array}$ & 2008 & 15 ans \\
\hline $\begin{array}{l}\text { Aménagement et exploitation du } \\
\text { Terminal à conteneurs de San Pedro } \\
\text { (TCS) }\end{array}$ & $\begin{array}{l}\text { Port Autonome de San } \\
\text { Pedro }\end{array}$ & $\begin{array}{l}\text { Mediterranean Shipping } \\
\text { Company } \\
\text { (MSC) }\end{array}$ & 2008 & 50 ans \\
\hline $\begin{array}{l}\text { Activité de remorquage de navires, } \\
\text { de veille sécurité remorqueur et de } \\
\text { lamanage }\end{array}$ & $\begin{array}{l}\text { - Port Autonome } \\
\text { d'Abidjan ; } \\
\text { - Port Autonome de San } \\
\text { Pedro }\end{array}$ & $\begin{array}{l}\text { Ivoirienne de Remorquage et de } \\
\text { Sauvetage } \\
\text { (IRES) }\end{array}$ & 2012 & 15 ans \\
\hline $\begin{array}{l}\text { Réalisation et exploitation du } \\
\text { Système de sureté au port d'Abidjan }\end{array}$ & $\begin{array}{l}\text { Port Autonome } \\
\text { d'Abidjan }\end{array}$ & Port sécurité Sarl & 2012 & 15 ans \\
\hline $\begin{array}{l}\text { Deuxième terminal à conteneurs } \\
\text { (TC2) d'Abidjan }\end{array}$ & $\begin{array}{l}\text { Port Autonome } \\
\text { d'Abidjan }\end{array}$ & Côte d'Ivoire Terminal & 2013 & 21 ans \\
\hline $\begin{array}{l}\text { Service de pilotage du port de San } \\
\text { Pedro }\end{array}$ & $\begin{array}{l}\text { Port Autonome de San } \\
\text { Pedro }\end{array}$ & Petrusienne & - & - \\
\hline
\end{tabular}




\subsection{Impact de la présence des acteurs privés dans le développement des ports ivoiriens}

- Une contribution à la modernisation des infrastructures et équipements portuaires

L'essor de la conteneurisation et la possibilité de massification qu'il offre a conduit les armateurs, grâce aux prouesses technologiques, à repousser sans cesse les limites des capacités d'emport des navires porte-conteneurs. L'industrie maritime s'est entièrement remodelée autour du conteneur. Ainsi, les ports ont dû adapter leurs installations pour faire face au gigantisme naval qui s'en est suivi, sous peine d'être marginalisées par les armateurs qui souhaitent réaliser des économies d'échelle. Cette exigence en vue de l'adaptation des installations des ports d'Abidjan et de San Pedro aux normes internationales, fait appel à une importante masse monétaire à laquelle l'État de Côte d'Ivoire à lui seul ne peut prétendre apporter des réponses efficientes. C'est alors que l'Etat, encouragé par les instances internationales, va faire appel aux opérateurs privés pour le soutenir dans son action de développement portuaire.

Ces accords de partenariats seront matérialisés par la signature de conventions de concessions pour la construction et l'exploitation d'infrastructures et d'équipements portuaires modernes. Le terminal à conteneurs de Vridi qui a fait l'objet du premier contrat de concession a vu ses capacités techniques renforcées. Le nombre de portiques (photo 1) dont disposait cette plateforme est passée de deux (avant libéralisation) à six. Les chariots élévateurs pour le stockage des conteneurs sur parc ont été remplacés par seize portiques de parc de dernière génération. La qualité des services connait une amélioration grâce au savoir-faire des opérateurs privés et au renforcement des équipements de manutention. Outre les redevances versées aux ports autonomes d'Abidjan et de San Pedro (que nous analyserons en aval) en contrepartie de l'exclusivité de l'exploitation des terminaux cédés, les concessionnaires, suivant les cahiers de charge, doivent respecter des obligations, dont certaines sont ci-après énumérées :

- exploiter, utiliser, réhabiliter, modifier, consolider, améliorer les biens constituants les ouvrages concédés selon les programmes d'investissement ;

- acquérir des biens de reprise, des biens propres et tous autres biens. Exploiter ces biens, le renouveler si nécessaire, les modifier, les compléter, les consolider ou les améliorer ;

- posséder et exploiter des installations pour la réparation des biens de la concession;

- développer le trafic maritime des terminaux à conteneurs afin de renforcer les capacités opérationnelles des ports d'Abidjan et de San Pedro, d'améliorer la qualité des services offerts aux usagers desdits 
ports, de développer le trafic de transbordement et de pratiquer des tarifs compétitifs pour les services rendus aux usagers.

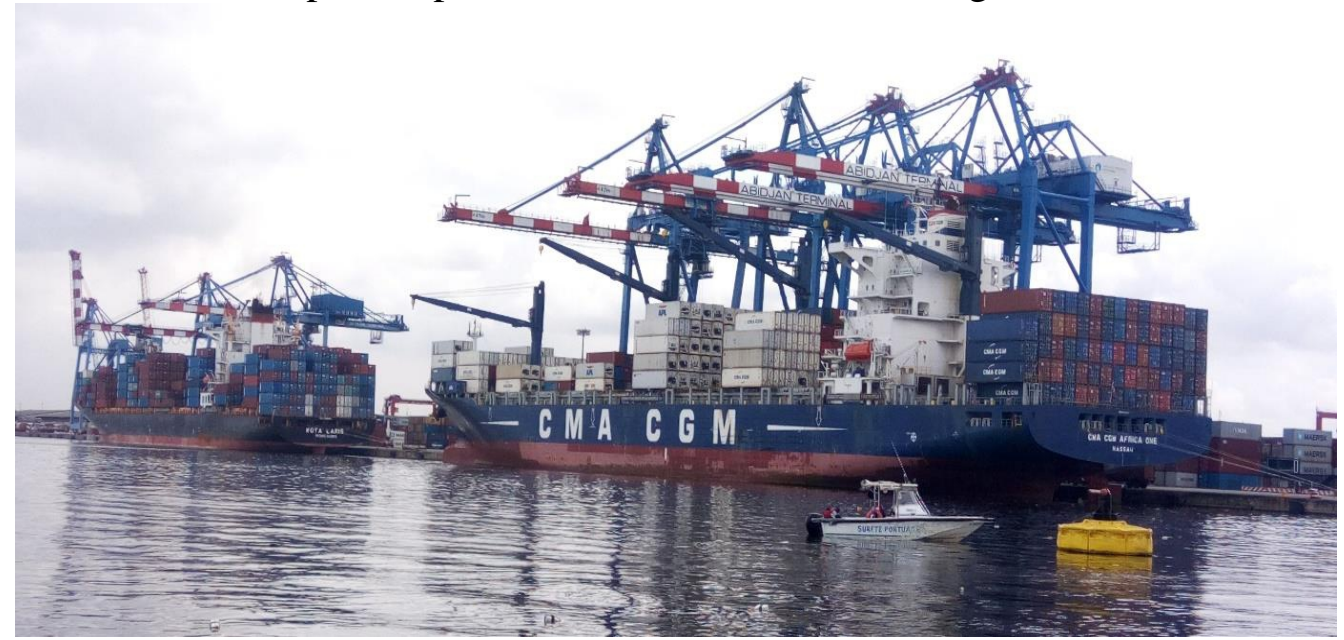

Photo 1: Une vue du terminal à conteneurs d'Abidjan avec en arrière-plan ses portiques de quai (Cliché: Mouto, 2018)

De vastes projets d'extension et de modernisation sont en cours dans les ports ivoiriens. À Abidjan, il s'agit de la construction du second terminal à conteneurs dénommé TC2 dont l'appel d'offre a été remporté par le consortium APM Terminal/Bolloré Africa Logistics/Bouygues Travaux Publics, des travaux de modernisation du port, ceux de l'élargissement et de l'approfondissement du canal de Vridi qui vient de s'achever (photo 2). Les travaux pour la réalisation de ce dernier projet est le fruit d'un partenariat public-privé. Il a bénéficié d'un financement à hauteur de $85 \%$ du coût global - 560 milliards de FCFA - d'Eximbank de Chine.

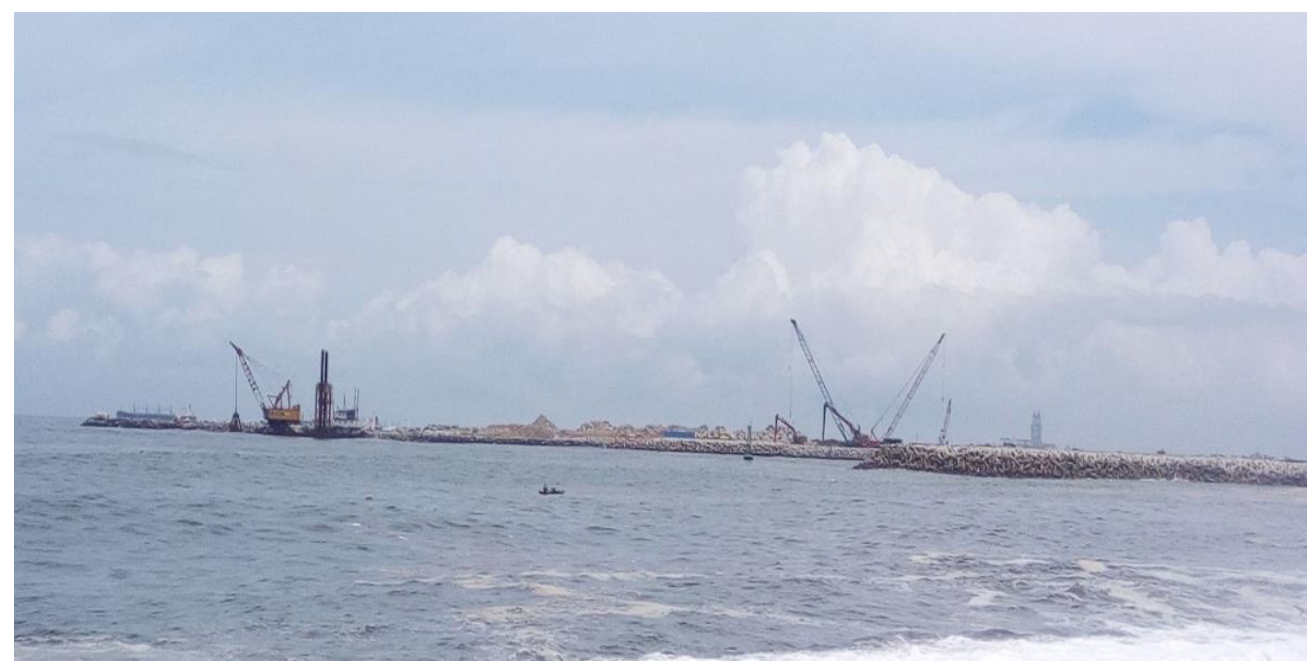

Photo 2: Les travaux d'élargissement et approfondissement du canal de Vridi (Cliché : Mouto, 2018) 
Du côté de San Pedro, les choses évoluent dans la même dynamique. Le groupe italo-suisse MSC a remporté en 2008, pour une durée de 35 ans, l'appel d'offre pour la création et l'exploitation d'un terminal à conteneurs et la construction des voies d'accès au port. À terme, la gestion de ces équipements et infrastructures sera confiée à la puissance publique à travers les représentants des autorités portuaires. Sauf au cas où les PAA et PASP voudraient les remettre en concession à nouveau. En attendant, les capitaux apportés par le secteur privé contribuent indéniablement à la modernisation des équipements et infrastructures des ports d'Abidjan et San Pedro ainsi qu'à leur développement.

- Un accroissement de la productivité et une amélioration de l'offre de service portuaire à Abidjan et à San Pedro

Des nombreux critères qui permettent à un chargeur ou un armateur de faire le choix d'un port au détriment d'un autre pour ses opérations commerciales figure la productivité de celui-ci. La productivité est rendue possible grâce aux équipements de pointe et au savoir-faire mis à disposition par les prestataires de services. Les indicateurs qui aident à évaluer cette productivité sont, entre autres, l'amélioration de la cadence de manutention (tableau 4), le temps d'attente en rade - 3 heures au maximum au Port d'Abidjan contre 24 heures au minimum dans les autres ports (CBC, 2019) ${ }^{3}$ et le temps de séjour à quai. Cette dernière variable varie selon le type de navire, sa taille et la nature de la cargaison qu'il transporte. Il est également fonction de la performance des équipements de manutention disponible dans le port d'accueil. Ainsi, selon qu'il s'agisse d'un navire porte-conteneurs de 4500 $\mathrm{EVP}^{4}$, d'un roulier de 24000 DWT, d'un navire cargos de 13000 DWT ou d'un vraquier de 56000 DWT, la durée d'attente au port d'Abidjan sera respectivement de 5 heures, 7 heures, 2,6 jours et 1,3 jours (Port autonome d'Abidjan, 2014, p70). Ce critère est déterminant tant pour le chargeur que pour l'armateur dans leur choix d'opérer dans un port. Sur une rangée portuaire ouest-africaine où la bataille entre les ports pour jouer le rôle de hub est ardue, l'amélioration de la qualité des services proposés par un accroissement de la productivité se présente comme un atout certain pour l'attractivité des ports ivoiriens. Elle pourrait avoir également des retombés économiques car un port qui traite avec célérité les navires est plus attractif pour les armateurs qui souhaitent rester le moins possible à quai.

${ }^{3}$ Site officiel du Conseil burkinabé des chargeurs à Abidjan http://www.cbc.bf/index.php/les-ports/cote-d-ivoire-abidjan

${ }^{4}$ Équivalent vingt pieds 
Tableau 4 : Évolution des cadences de manutention du terminal à conteneurs du port d'Abidjan

\begin{tabular}{|l|c|c|c|c|c|c|c|c|c|}
\hline $\begin{array}{c}\text { Rendement } \\
\text { (Mvts/h) }\end{array}$ & 2002 & 2003 & 2004 & 2006 & 2007 & 2008 & 2009 & 2010 & 2011 \\
\hline Portiques & 12 & 12 & 18.3 & 21.3 & 23,3 & 23,1 & 21,1 & 19,8 & 20,6 \\
\hline Grues & 13 & 14 & 18 & 17,5 & 19,5 & 19,5 & 17,4 & 17,0 & 15,9 \\
\hline Global & 13 & 12 & 18,2 & 20,6 & 22,5 & 22,3 & 20,2 & 19,2 & 19,4 \\
\hline
\end{tabular}

Source: PAA/DEEP-DEV/DPP, 2012

On observe une nette amélioration des performances des portiques et des grues entre la période d'avant la gestion du terminal par le consortium Bolloré/APM-Terminal (2003) et la période qui se situe juste après cette date. Les performances des équipements du terminal se sont constamment accrues entre 2004 et 2011, passant respectivement de 18,3 et 18 mouvements par heure $(\mathrm{Mvts} / \mathrm{h})$ à 20,6 et 15,9 Mvts/h pour les portiques et les grues.

- Une augmentation des recettes portuaires versus une baisse des coûts d'investissement publics

L'amélioration de l'efficience des terminaux et services concédés a indubitablement entrainé une hausse des trafics de tous genres et par ricochet, une augmentation des recettes portuaires. En effet, il n'est plus à démontrer qu'un port efficace exerce une forte attractivité sur les armateurs et chargeurs et génère naturellement un volume de trafic plus important. L'explosion du trafic global du port de San Pedro qui est passé de moins de 1 million de tonnes de marchandises traitées annuellement à plus de 4 millions après la mise en concession de son terminal à conteneurs en 2008 vient soutenir cette assertion (Port Autonome de San Pedro, 2015; p17). La figure 1 ci-après présente l'évolution du trafic de conteneurs au port de San Pedro de 1978 à 2015. En parlant des différentes concessions portuaires accordées à Abidjan et à San Pedro, il faut noter qu'en contrepartie des droits qui leurs sont transférés, les concessionnaires sont tenus de payer deux types de redevances: des redevances fixes et des redevances variables. Qu'elles soient fixes ou variables, les redevances dépendent de l'objet de la concession. Deux exemples serviront de bases d'illustration dans cette analyse. Il s'agit des conventions de concession du premier terminal à conteneurs du port d'Abidjan et la convention de concession de l'activité de remorquage de navires, de veille sécurité remorquage et de lamanage. La dernière convention s'étend au port de San Pedro. 
Figure 1 : Évolution du trafic de conteneurs au port de San Pedro de 1978 à 2015 400000

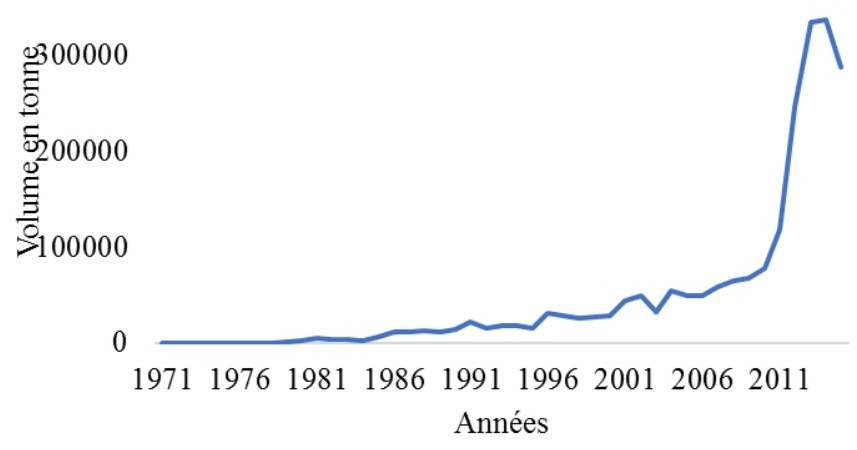

Source: Statistiques - Port Autonome de San Pedro, rapports d'activités PASP, 2014 et 2015

Nul entre 1971 et 1978, le trafic de conteneurs au port de San Pedro a commencé timidement avec 1770 EVP en 1979. En 2004, ce trafic a franchi pour la première fois la barre des $50000 \mathrm{EVP}$ avec un trafic de $54540 \mathrm{EVP}$ traités. Mais c'est après la concession du terminal à conteneurs à l'opérateur MSC en 2008 que le trafic de conteneurs au port de San Pedro a connu une envolée. À partir de cette date le trafic de conteneurs dans ce port a enregistré une croissance positive pour atteindre le chiffre record de 336767 EVP en 2014. Cela traduit le dynamisme impulsé par MSC à cette activité depuis sa prise en main.

Ainsi, relativement à la concession du premier terminal à conteneurs d'Abidjan dénommé Abidjan Terminal (ATL), le concessionnaire paie au PAA une redevance annuelle fixe de vingt-deux Euros (22 Euros) par mètre carré calculée sur la superficie des terrains mis à disposition. Or, la superficie du domaine exploité par ATL correspond à 30 ha 26 a $31 \mathrm{ca}$, soit $302631 \mathrm{~m}^{2}$ ce qui donne un montant de 6657882 Euros soit 4367284 303, 074 FCFA à payer par an par ATL comme redevance annuelle fixe. Quant à la redevance variable payable par ATL au PAA, elle est calculée selon la formule suivante :

a) Dix Euros (10 Euros) par EVP hors transbordement si le trafic reste supérieur à trois cent mille (300 000) EVP

b) Quatre Euros (4 Euros) par EVP pour les conteneurs en transbordement

La figure 2 suivante montre l'évolution des redevances perçues par le PAA sur la concession d'ATL de 2004 à 2015. 
Figure 2 : Évolution des redevances du Terminal à conteneurs d'Abidjan de 2004 à 2015

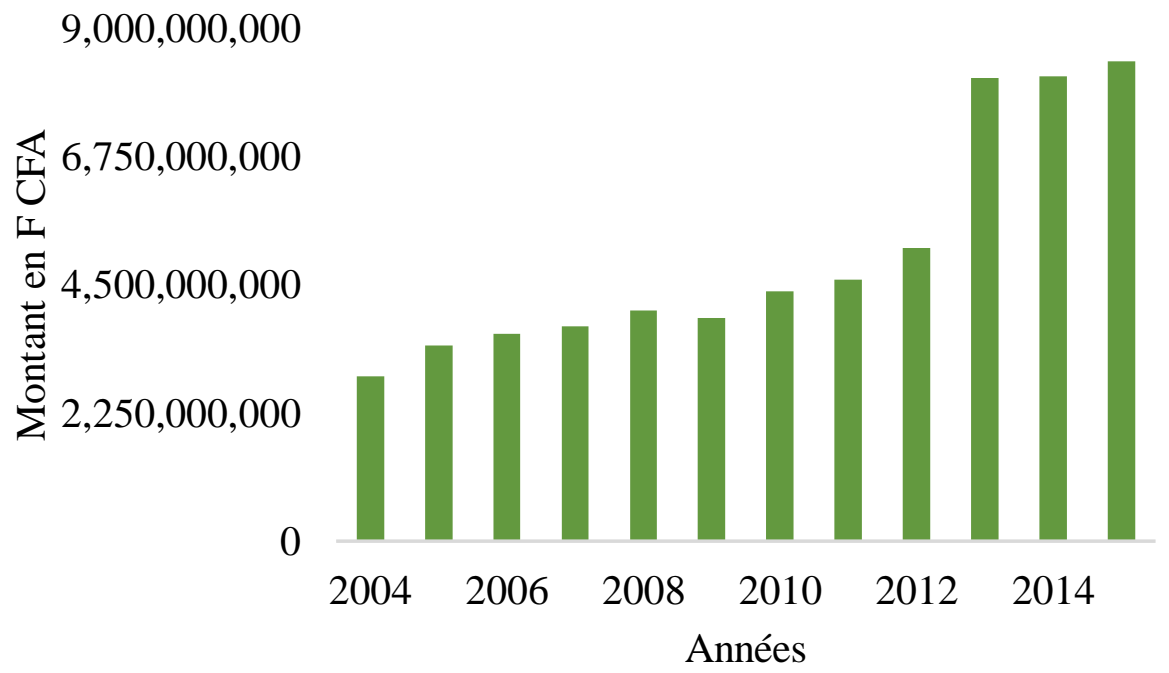

Source: Direction du Contrôle, de l'Audit, et de la Qualité -Port Autonome d'Abidjan, 2017

Les redevances perçues par le Port Autonome d'Abidjan en contrepartie de l'exploitation du terminal à conteneurs de Vridi ont globalement augmenté. De 2882264862 F CFA en 2004, ces redevances représentaient $5147998855 \mathrm{~F}$ CFA en 2012. À partir de 2013, le Port Autonome d'Abidjan a perçu 8127442756 F CFA en guise de droit sur l'exploitation du terminal de Vridi. La variation d'environ $3000000000 \mathrm{~F}$ CFA entre 2012 et 2013 fait suite à un audit réalisé par la Direction du Contrôle, de l'Audit, et de la Qualité du Port Autonome d'Abidjan qui a dut revoir les montants de la redevance payé par le concessionnaire. En 2015, la redevance payée par ATL en échange du droit d'exploitation du terminal de Vridi était de 8418996855 F CFA. La croissance positive des tarifs de la redevance sur concession du terminal d'Abidjan est inversement proportionnelle au volume de conteneurs transbordés. L'augmentation des redevances perçues malgré la baisse du trafic en transbordement pourrait signifier que le taux de conteneurisation de marchandises destinées au marché local ivoirien est en hausse. Quant à la chute du taux de marchandises transbordées à Abidjan, elle traduit la baisse de compétitivité du port d'Abidjan par rapport aux ports voisins de la COA, Lomé notamment qui capte un trafic important de navires en provenance d'Asie.

S'agissant de la convention de concession du service de remorquage de navires, de veille sécurité remorquage et de lamanage à l'Ivoirienne de Remorquage et de Sauvetage (IRES), deux types de droits sont perçus par les autorités concédantes (PAA et PASP). Il s'agit des droits de concession forfaitaires - de 1494214 euros pour le PAA et de 136991 euros pour le 
PASP - et des redevances de concessions. Les redevances de concessions sont composées de :

a) la redevance d'exploitation pour le service de remorquage navire dans les ports d'Abidjan et de San Pedro ;

b) la redevance d'exploitation pour la veille sécurité pour le port d'Abidjan ;

c) et la redevance d'exploitation pour le service de lamanage pour le port d'Abidjan.

La redevance d'exploitation elle-même est composée de deux parties : une partie fixe et une partie variable qui sont liées aux opérations effectuées. L'assiette de calcul de la partie variable est le volume du navire défini comme suit:

$\mathrm{V}=\mathrm{L} \times \mathrm{b} \times \mathrm{Te}$ où $\mathrm{V}$ est exprimé en mètre cube, $\mathrm{L}, \mathrm{b}$ et Te représentent respectivement la longueur hors tout du navire, sa largeur maximale et son tirant d'eau maximal.

- Remorquage navires au port d'Abidjan et San Pedro

Redevance d'exploitation navire au port d'Abidjan :

- Partie fixe : 147449 Euros, soit 96663 140,93 FCFA

- Partie variable: $\mathrm{R}=\mathrm{V}$ x Nombre de remorqueurs x 0,0008 Euros

Redevance d'exploitation navires port de San Pedro :

- Partie fixe : 16440 Euros, soit 10777 570,8 FCFA

- Partie variable: $\mathrm{R}=\mathrm{V}$ x Nombre de remorqueurs x 0,0008 Euros

- Veille sécurité remorqueur au port d'Abidjan

Redevance veille sécurité remorqueur :

- Partie fixe : 19050 Euros, soit 12488 608,5 FCFA

- Partie variable: $\mathrm{R}=\mathrm{V}$ x Nombre de remorqueurs x 0,0004 Euros/heure de sécurité

$\mathrm{Au}$ regard de tout ce qui précède, il est clairement établi que le partenariat avec le secteur privé contribue fortement à l'augmentation des recettes des ports d'Abidjan et de San Pedro. Paradoxalement, à l'opposé des trente années de l'après indépendance ou l'État était le principal investisseur dans l'aménagement et l'équipement des ports, le relai a été passé au secteur privé à travers les contrats de concessions. Toutefois, l'État - par l'autorité portuaire - lorsqu'il doit participer au financement d'un projet, apporte une contribution généralement plus modeste que celle des partenaires privés. Il a perdu son statut de cheville ouvrière en termes d'investissement. Le secteur privé est à l'avenir inscrit au premier rang des investisseurs pour la modernisation et le développement des ports ivoiriens. Les acteurs privés sont des partenaires incontournables. Les types de partenariats envisagés pour les projets de développement portuaire récemment réalisés, en cours de réalisation 
ou en attente d'être réalisés à Abidjan et à San Pedro sont des concessions de type BOT ou des partenariats public et privé (PPP) avec une forte participation du secteur privé à l'image du projet de modernisation du port d'Abidjan financé à hauteur de $85 \%$ par une banque chinoise.

- Le risque d'un transfert du monopole public à un monopole privé

La coopération entre acteurs publics et privés est d'un intérêt particulier pour le développement des ports d'Abidjan et de San Pedro. Pourtant, cette collaboration suscite des craintes quant au transfert d'un monopole du marché naguère détenu par le secteur public au secteur privé. Il est encore plus à craindre que cette situation monopolistique soit profitable non pas à l'ensemble des acteurs privés, mais seulement à une poignée d'entre eux. Or, l'une des raisons ayant conduit la Banque Mondiale et les autres bailleurs de fonds à inciter la Côte d'Ivoire à reformer sa politique maritime et portuaire était justement l'idée de briser le monopole de l'État dans la gestion des affaires maritimes et des opérations portuaires. Privilège qui, selon ces institutions financières, ne favorisait pas la concurrence dans ce milieu et influait négativement sur la compétitivité des produits made in Côte d'Ivoire sur les marchés internationaux. Le spectacle auquel il est donné d'assister depuis la libéralisation du transport maritime est l'arrivée massive d'opérateurs privés aux ports d'Abidjan et San Pedro. Cette diversité d'opérateurs portuaires est d'ailleurs une bonne chose, car elle offre un large éventail de choix aux chargeurs et opérateurs économiques.

Par contre, là où réside la difficulté c'est que plus de la moitié des trafics réalisés dans ces deux ports est le fait d'une poignée de ces acteurs privés. De plus, la quasi-totalité des conventions de concessions signées entre les représentants des autorités portuaires - PAA et PASP - est restreint à un petit groupe d'acteurs privés. Pire, certains opérateurs privés comme Bolloré sont partie prenante dans plusieurs contrats de concessions avec des contrats pour une même activité - ATL et TC2 - au port d'Abidjan. Cet état de fait est une entrave au jeu de la concurrence qui est une raison ayant présidé à la libéralisation. Le processus de cession au secteur privé affecte toutes les activités et services desquels l'autorité portuaire se désengage. Même le pilotage et la sûreté portuaire qui apparaissaient comme des fonctions hautement régaliennes n'y échappent pas. Enfin, l'occupation puis l'exploitation des terminaux par des groupes privés n'a pas que des aspects positifs ; elle a aussi des revers. L'appartenance de MSC - transporteur maritime et opérateur du terminal conteneurs du port de San Pedro - et Bolloré - opérateurs de Abidjan Terminal et du TC2 et de surcroit, partie prenante dans les autres services de pré et post acheminement - aux groupes prestataires de services puis à ceux de demandeurs de services par exemple, enfreint les règles de base de la concurrence. De manière concrète, MSC possède ses propres navires et Bolloré est le consignataire de plusieurs compagnies 
maritimes. Lorsque leurs navires arrivent à San Pedro ou à Abidjan (selon qu'il s'agisse de MSC ou de Bolloré), la priorité leur est accordée dans les terminaux dont ils sont les gestionnaires. Ce malgré la règle qui prévaut dans les deux ports qui stipule que le premier arrivé est le premier servi. C'est tout naturellement qu'après les opérations portuaires ces sociétés confient les opérations de transport terrestre à leurs structures de transit. Ces grandes sociétés offrent un service intégré capable d'assurer le transport de bout-enbout de la marchandise.

- Les impacts géopolitiques de la libéralisation du transport maritime en Côte d'Ivoire

Depuis presque deux décennies la côte Ouest africaine est le théâtre d'une bataille entre les ports de la région pour se positionner en tant que principal port d'éclatement. Le défi que s'est fixé chaque port pour réaliser cette ambition est de gagner le pari du port le plus développé à travers la qualité des équipements, infrastructures, la nature et la qualité des prestations proposées, etc. La réalisation de ces projets de développement portuaire demande de gros financements que seuls les opérateurs privés sont à mesure de décaisser. C'est cette stratégie qui a permis aux opérateurs à l'image de Bolloré de prendre progressivement le contrôle de plusieurs terminaux dans différents ports de la rangée.

En l'absence du secteur public dans les opérations d'exploitation portuaire - en plus de son absence sur les océans - les opérateurs privés, présents dans quasiment tous les ports sont les seuls acteurs susceptibles de définir un port de la région en tant que $h u b$ selon une logique qui leur est propre. Autrement, faire d'Abidjan ou de San Pedro le principal port d'éclatement de la côte Ouest-africaine ne dépend plus uniquement de leurs autorités portuaires. Cet ambitieux projet relève avant tout de la volonté des grandes sociétés maritimes et des opérateurs multiports comme Bolloré, APM terminal, Maersk, MSC, ...

\section{L'incidence des Activites des Compagnies Maritimes Sur l'economie Portuaire de la Cote d'Ivoire}

Les sociétés maritimes jouent un rôle déterminant dans le développement de l'économie portuaire de la Côte d'Ivoire. Elles sont inscrites en première ligne de l'activité maritime et portuaire. Les effets positifs liés aux activités de ces compagnies sont mesurables à plusieurs niveaux. Ces actions portent sur les diverses redevances perçues par les ports autonomes d'Abidjan et de San Pedro, à la création de nombreux emplois. Enfin, elles permettent l'intégration de la Côte d'Ivoire à la mondialisation par son interconnexion aux autres continents via les nombreuses offres maritimes qu'elles proposent. 


\subsection{Les sociétés maritimes, principaux acteurs du développement de I 'économie portuaire ivoirien}

Les rôles économiques tenus par les sociétés maritimes présentes dans les ports ivoiriens ne se résolvent pas au seul fait de débarquer ou d'embarquer des marchandises bien que cette opération en elle-même soit source d'une importante manne financière pour les ports autonomes d'Abidjan et de San Pedro. Les devises tirées des sociétés maritimes proviennent aussi des différentes redevances perçues sur les navires qui entrent et sortent des ports, sur les marchandises qui y sont embarquées ou débarquées. Des redevances sont également perçues sur l'occupation du domaine portuaire par les sociétés maritimes ou de toute autre structure dont les activités sont liées directement ou indirectement à celle des sociétés maritimes. Enfin, des redevances sont prélevées des sociétés maritimes pour l'usage des installations portuaires. À partir des types de redevances perçues par les ports autonomes d'Abidjan et de San Pedro, l'on peut se rendre compte du rôle économique déterminant joué par les sociétés maritimes dans lesdits ports (figure 3).

Figure 3: Évolution des revenus procurés par les ports d'Abidjan et San Pedro de 2010 à 2015

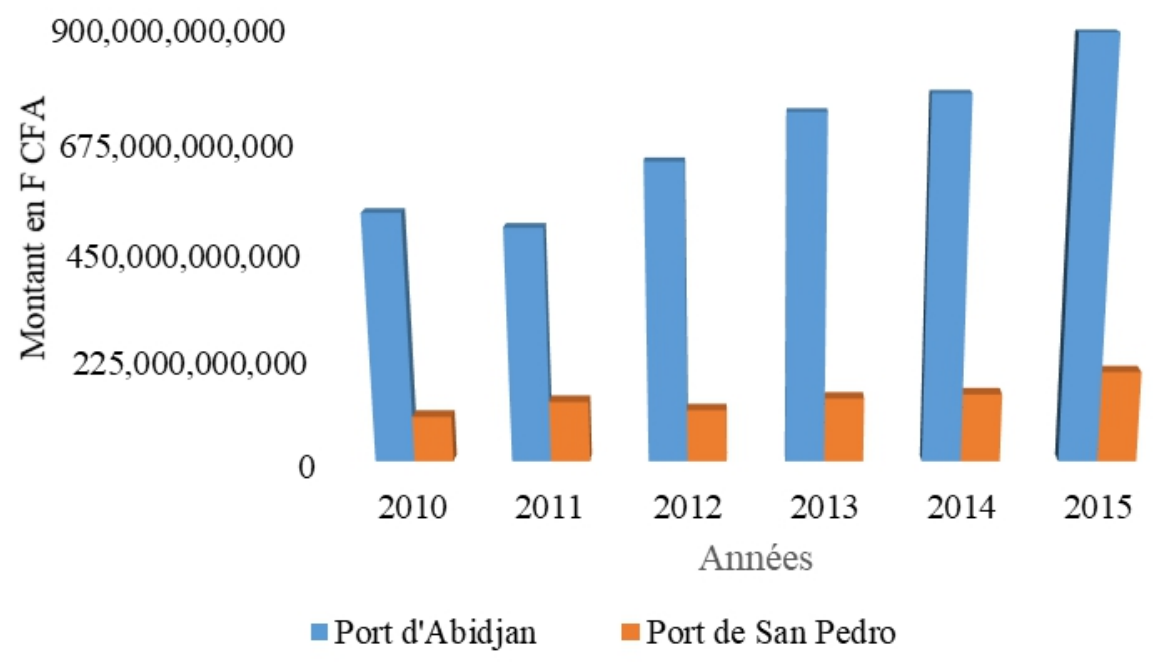

Source: Direction générale des Douanes, 2017

Le revenu procuré par les ports d'Abidjan et San Pedro ont connu une constante évolution entre 2010 et 2015. De 529313273178 F CFA pour le port d'Abidjan et 97266816549 F CFA pour le port de San Pedro en 2010, ces chiffres sont respectivement passés à 889397673948 F CFA et 194718694118 F CFA en 2015. Cette évolution traduit le dynamisme des ports ivoiriens et prouve que ces deux entités attirent de nombreux opérateurs maritimes et portuaires qui contribuent par ailleurs, à travers les redevances qu'elles paient, au développement de l'économie portuaire de la Côte d'Ivoire. 
En somme, les redevances imposées aux sociétés maritimes dans les ports ivoiriens sont les redevances sur les navires, les redevances sur conteneurs, les redevances sur les marchandises et pour terminer les redevances d'occupation du domaine portuaire.

\subsection{Les compagnies maritimes, un facteur essentiel de l'intégration de la Côte d'Ivoire dans la mondialisation}

Les continents, bien qu'étant séparés les uns des autres par les océans, sont unifiés par les routes transocéaniques qui les relient. Et par conséquent, les transports maritimes permettent la circulation d'importants flux de marchandises sur ces axes. La globalisation des échanges qui en résulte veut que les lieux de production soient de plus en plus éloignés des lieux de consommation finaux. Ainsi, une grande partie de ce que nous consommons transite par la mer. Les flux maritimes assurent (en volume) $90 \%$ du commerce mondial (Couvenhes, 2013).

La Côte d'Ivoire est un pays à économie extravertie. Elle exporte ses productions pour l'essentiel agricoles et importe des produits manufacturés et des produits industriels de base nécessaire pour approvisionner ses marchés locaux et ses unités industrielles. Les échanges extérieurs de la Côte d'Ivoire s'effectuent par la mer. Et les ports d'Abidjan et de San Pedro sont des plateformes par lesquelles transitent plus de $90 \%$ de ce commerce extérieur de la Côte d'Ivoire (Tapé, 2004). Les compagnies maritimes sont les principaux moteurs de l'intégration de la Côte d'Ivoire au processus de la mondialisation, en lui permettant d'acquérir des marchandises à l'étranger utiles pour sa population et de placer par la même occasion ceux qu'elle produit sur les marchés internationaux.

\section{Discussion}

Les ports ivoiriens sont gangrénés par de nombreux obstacles qui contrarient sérieusement leur développement. Afin de pallier ces problèmes, des réformes institutionnelles et l'ouverture des opérations maritimes et portuaires aux partenaires privés se sont avérées indispensables. Cette mesure en faveur des acteurs privés notamment les opérateurs multiports a permis aux ports d'Abidjan et de San Pedro de bénéficier du savoir-faire de ces nouveaux partenaires en matière de gestion, de contacts, de modernisation des équipements et d'apports de fonds pour la réalisation des infrastructures. Ces résultats sont conforment à ceux de la Banque mondiale qui estime que pour la seule année 2000, les investissements du secteur privé dans les projets portuaires dans les pays en développement ont été de 2 milliards 632 millions de dollars E-U, contre 304 millions de dollars E-U en 1992. L'instance financière internationale montre ainsi le rôle primordial que peuvent jouer les acteurs privés dans le processus de développement des ports de façon générale 
et plus encore dans celui des ports africains. Le partenariat avec le secteur privé, laisse comprendre LA CNUCED (2003 ; P6), assure l'amélioration de l'efficacité des services ainsi que l'entretien, le renouvellement et la performance des équipements. Ainsi, selon le même rapport les principales conséquences de la coopération entre le secteur privé et le secteur public sont essentiellement l'amélioration de la performance des opérations, l'augmentation du trafic, la diminution des charges portuaires, etc. Pour le secrétariat de la CNUCED dans son rapport de 2003 (p10) la coopération avec le secteur privé est de plus en plus valorisée sur le continent africain afin d'obtenir des ressources financières complémentaires pour moderniser les ports et de profiter des expériences des entreprises privées, essentiellement étrangères, en matière de gestion et de productivité.

Les différentes situations décrites plus haut accordent une place cruciale aux acteurs privés dans les scénarios de modernisation des ports en Afrique. Cependant, le succès d'un port relève-t-il du seul fait des opérateurs privés ? A ce propos, Brenda (2009; p1) nous apporte un éclairage. Selon cet auteur, depuis l'an 2000, le port de Tema a effectué une spectaculaire remontée et se classe maintenant parmi les trois ports commerciaux les plus importants d 'Afrique subsaharienne. Une part de ce succès peut être attribuée à l'implication des géants habituels du secteur. Toutefois, plus qu'à un processus classique de privatisation, Tema doit sa réussite à l'engagement d'une institution étatique, la Ghana Portsand Harbours Authority. A travers cet exemple, l'auteur met en avant l'importance du rôle des autorités publiques dans la politique de développement portuaire au Ghana sans toutefois remettre en cause la nécessité de sceller une alliance entre le secteur public et le secteur privé.

Certes, le but de la libéralisation est de contribuer au développement des ports mais au préalable elle a favorisé le passage d'un système de gestion à un autre. C'est d'ailleurs ce qui fait dire à Souhir et Jacques (2008; p23) que la libéralisation a permis de passer d'une structure monopolistique à une forme de concurrence où le secteur privé est plus impliqué dans le processus opérationnel et dans l'investissement. L'objectif visé par ce changement dans la gestion des ports consiste bien à améliorer l'efficience portuaire par une concurrence accrue sous la férule d'un régulateur indépendant respectueux de l'équilibre privé-public poursuivent-ils.

Le transfert de compétences des acteurs publics à leurs partenaires privés n'a pas que des aspects positifs. Il confère à certains opérateurs privés notamment les grands groupes multiports une position dominante qu'il n'hésite pas parfois à abuser. C'est le cas avec le groupe Bolloré à travers sa filiale Bolloré Africa Logistic présent dans presque tous les ports de la côte Ouest et centre africaine. L'hégémonie des grandes structures privées dans l'exploitation des ports interroge sur les dangers réels du partenariat public- 
privé. C'est pourquoi dans son rapport de 2003; p1, le secrétariat de la CNUCED, compte tenu de la limite des trafics de la plupart des ports africains et du risque de transfert d'un monopole public à un monopole privé, estime que l'option dite "port propriétaire foncier" (landlord port) est pertinente dans le cadre du partenariat public-privé pour la gestion des port en Afrique.

Les ports doivent-ils être gérés par des organismes de droit privé et sont-ils soumis à la concurrence, au risque de créer des situations de position dominante ? N'y a-t-il pas là une remise en cause de l'intérêt général et du service public portuaire et donc du pouvoir régalien, le port étant un élément de développement économique principal pour un Etat ? S'interroge GUERLET (2013; p20).

\section{Conclusion}

La présente étude a montré qu'avant la libéralisation du transport maritime l'Etat jouait un rôle crucial dans la gestion, l'aménagement et le processus de développement des ports ivoiriens. Le modèle de port-service dans lequel le rôle de premier plan lui était dévolu était la trajectoire de gouvernance des ports d'Abidjan et de San Pedro. Cette politique du « tout par l'Etat » a eu de nombreux avantages, notamment pour la maîtrise du coût du fret indispensable pour la compétitivité des produits made in Côte d'Ivoire sur les marchés internationaux. Mais elle a aussitôt montré ses limites par l'incapacité de l'Etat ivoirien à répondre aux nouvelles exigences du transport maritime en termes de modernisation et d'efficacité. Dès lors, le soutien du secteur privé est devenu indispensable et le développement d'un partenariat public-privé plus qu'une nécessité.

La libéralisation du transport maritime en Côte d'Ivoire a fait des zones portuaires d'Abidjan et de San Pedro des terreaux propices à l'installation de nombreuses sociétés privées spécialisées dans les activités maritimes et portuaires. Ces derniers, au gré des reforme et par des contrats de concession ont substitué le secteur public dans son rôle de principal exploitant portuaire. Dorénavant, les opérateurs privés sont des pions clés sur lesquels repose le processus de développement des ports ivoiriens comme en témoigne leurs prises de participations dans de nombreux projets de modernisation portuaire aussi bien à Abidjan qu'à San Pedro. Cependant, si la libéralisation du transport maritime en Côte d'Ivoire est à la base de nombreux effets positifs, cette politique interroge toutefois sur le risque d'un passage du monopole public à celui d'un monopole privé par l'action d'une poignée d'opérateurs multinationaux.

\section{References:}

1. Abbes, S., et Guillaume, J. (2008). Chapitre 9. Ports de commerce et économie portuaire. In Guillotreau, P. (Ed.), Mare economicum : 
Enjeux et avenir de la France maritime et littorale. Presses universitaires de Rennes. p. 361-38. doi : 10.4000/books.pur.53818

2. Brenda C. (2009). « La rénovation du port de Tema - Économie politique de la frontière maritime du Ghana », in Politique africaine, $\mathrm{N}^{\circ} 116, \mathrm{p} 63$ à 84

3. CNUCED, 2003, « Réformes et place du secteur privé dans les ports africains », In: Rapport du secrétariat de la CNUCED, Genèvre, CNUCED, [en ligne], 31p. http://unctad.org/fr/docs/sdtetlb5_fr.pdf,

4. Couvenhes, M. F. (2013). La mer au cœur de la mondialisation des échanges. Rennes - journée académique du 2 octobre - enseigner la mer. Retrieved from http://espaceeducatif.acrennes.fr/jahia/webdav/site/espaceeducatif3/users/cmarechal1/public/ G\%C3\%A9ographie/Ressources/La\%20mer\%20au\%20c\%C5\%93ur $\% 20 \mathrm{de} \% 201 \mathrm{a} \% 20$ mondialisation $\% 20 \mathrm{des} \% 20 \% \mathrm{C} 3 \%$ A9changes $\% 20$ $\% 20$ expos\%C3\%A9.pdf

5. De Matons J. G. (Ed), 2012, Les concessions portuaires. Caen (France), Cormelles-le-Royal: EMS, 295 p

6. Gueguen-hallouë T. G., 2014, « Le rôle croissant du secteur privé dans l'activité et la gestion des ports », in Questions internationales, $\mathrm{n}^{\circ} 70$, France, Dossier: les grands ports mondiaux, La Documentation française, p. 65-77.

7. Guerlet, G. (2013). La gestion des ports par une entité publique: aspects européens et environnementaux, thèse de Doctorat, Université du Littoral d'Opale, 289p

8. Guessan, B. G. E. (2004). Effets de la libéralisation des transports maritimes sur l'économie ivoirienne. DESS Haute Études en Gestion de la Politique Économique, $21 \mathrm{p}$

9. Hartmann O. (2010). «Comment les pays enclavés s'articulent-ils à la mondialisation? Ports et commerce en Afrique de l'Ouest », in Afrique contemporaine, Louvain-la-Neuve (Belgique) et Paris (France), De Boeck Supérieur, $\mathrm{N}^{\circ} 234$ (2), p. 41-58, [en ligne], DOI. 10.3917/afco234.0041., consulté le 11/12/2017

10. Jacques G. (2012), «Les mutations récentes de la gouvernance des ports français sous la pression des contraintes internationales », in L'Espace Politique [En ligne]. URL: http://journals.openedition.org/espacepolitique/2202; DOI: 10.4000/espacepolitique. 2202

11. Mouto G. M (2019), Libéralisation du transport maritime international et économie portuaire en Côte d'Ivoire, thèse unique de Doctorat, IGT, université Félix Houphouët-Boigny, Abidjan, 308p (en cours)

12. Osmont, A. (1996.) La Banque Mondiale et les villes: du développement à l'ajustement, Karthala, Paris, 390 p 
13. Port Autonome d'Abidjan, 2014, Étude de compétitivité du port d'Abidjan - Rapport final, Paris, INECOR, $185 \mathrm{p}$

14. Port Autonome de San Pedro. (2015). Rapport annuel. $36 \mathrm{p}$

15. Tapé B J. (2008), « Les échanges maritimes de la Côte d'Ivoire et les questions de trafic interportuaire avec la France de 2000 à 2004 », Anoh K P. et Pottier P (dir), Géographie du littoral de Côte d'Ivoire Élément de réflexion pour une politique de gestion intégrée, coopération interuniversitaire, Cocody - Abidjan (Côte d'Ivoire) / Nantes (France), p. 103-142

16. Tapé, B. J. (2004). Économie maritime et portuaire de Côte d'Ivoire: étude géographique. (Doctorat dissertation). Université de Cocody, Abidjan, $876 \mathrm{p}$.

17. Tourret P., \& Valero C., 2017, «Le développement de la conteneurisation, symbole de la modernisation des ports africains », in Secteur Privé \& Développement, Paris, Propaco, $N^{\circ} 26$, mars, avril, mai, p. 30-33, [en ligne], https://blog.secteur-privedeveloppement.fr/2017/05/22/le-developpement-de-laconteneurisation-symbole-de-la-modernisation-des-ports-africains/, consulté le 05/10/2018 